\title{
Dual Image Watermarking Algorithm with SVD-DWT and Edge Detection on Different Layers of Colored Image
}

\author{
Anjali Kathpal \\ Research Scholar, CSE Dept. \\ Shaheed Bhagat Singh State Technical Campus \\ Ferozepur, India
}

\author{
Sonika Jindal \\ Assistant Professor, CSE Dept. \\ Shaheed Bhagat Singh State Technical Campus \\ Ferozepur, India
}

\begin{abstract}
Digital image watermarking is an emerging copyright protection technology. This paper proposed a new robust digital image watermarking technique to protect the data. In this paper, Dual Watermarking Scheme based on DWT-SVD is presented to improve the robustness and protection. Both Discrete Wavelet Transform and Singular Value Decomposition have been used as a mathematical tool to embed watermark in the image. In this proposed technique, two watermarks are embedded on the different layers in the host image. Various techniques are proposed to hide the data but current technique shows robustness against various attacks.
\end{abstract}

\section{Keywords}

Watermarking, Discrete Wavelet Transform, Singular Value Decomposition, Edge Detection.

\section{INTRODUCTION}

The term 'digital watermarking' was first appeared in 1993, when Tirkel et al presented two watermarking techniques to hide the watermark data in the images. The digital watermarking is use to hide the proprietary information in multi-media by embedding an ownership data into given data. This ownership data is called watermark and the given data is called host data [1][2]. The watermark should be embedded into host data (image, audio or video) in such a way that it should not only robust against common attack but also against malicious attacks. Watermarking algorithm mainly consist of an embedding and an extraction algorithm [3]. The watermarking can be defined as the process of embedding information in media files for authentication purpose which can be extracted later on. Watermarking helps to prove the rightful possessor ship of the object. The watermark added can be either visible or invisible. The main issues in the watermarking scheme are that the watermark should not degrade the quality of image and should not be perceptual to human eye [4].

Broadly watermarking technique can be classified into two domains: Spatial domain and Transform domain. In spatial domain data is embedded directly by modifying pixel values of host image, while in transform domain data is embedded by modifying transform domain coefficients. Transform domain shows more robustness against various attacks so it is more preferred then spatial domain [3].

Nowadays, Singular Value Decomposition (SVD) is a new transform used for watermarking. Garauv bhatnagar et al. proposed a new semiblind reference watermarking scheme based on discrete wavelet transform and singular value decomposition for copyright protection and authenticity which with stand under various attacks [5]. Kapre et al. proposed robust image watermarking based on singular value decomposition and discrete wavelet transform which show robust against various geometrical attacks [6]. Praful et al. proposed DWT-SVD semi blind image watermarking high frequency band which is resistant against various attacks [7]. In this paper, a new robust digital watermarking technique is proposed.

In this paper, firstly edges of the original image is extracted to take it as first watermark and another image is taken as second watermark. Then these two watermarks are hide on different layers of image with the help of DWT-SVD. This paper is organized as follows in section 2 introduces Singular Value Decomposition, Edge Detection, Discrete Wavelet Transform, section 3 describes the Proposed Method, section 4 explains the experimental results and section $5 \& 6$ gives the conclusion and references.

\section{TERMINOLOGY}

\subsection{Singular Value Decomposition}

The Singular Value Decomposition is one of the most useful tools of linear algebra with several applications to multimedia. Applications including Image compression, Watermarking and other Signal Processing. Given a real matrix, A $(m, n) ; 1 \leq$ $\mathrm{m} \leq \mathrm{M}, 1 \leq \mathrm{n} \leq \mathrm{N}$, it can be decomposed into a product of three matrices given by equation (1)

$$
\mathrm{A}=\mathrm{USV}^{\mathrm{T}}
$$

Where $\mathrm{U}$ and $\mathrm{V}$ are orthogonal matrices, $\mathrm{U}^{\mathrm{T}} \mathrm{V}=\mathrm{I}, \mathrm{V}^{\mathrm{T}} \mathrm{V}=\mathrm{I}$, and $S=\operatorname{diag}(\lambda 1, \lambda 2, \ldots . \lambda \mathrm{r})$.

The diagonal entries of $\mathrm{S}$ are called the singular values of A, the columns of $U$ are called the left singular vectors of $A$, and the columns of $\mathrm{V}$ are called the right singular vectors of $\mathrm{A}$. This decomposition is known as the Singular Value Decomposition (SVD) of A, and can be written as shown in equation (2),

$$
\mathrm{A}=\lambda 1 \mathrm{U} 1 \mathrm{~V} 1^{\mathrm{T}}+\lambda 2 \mathrm{U} 2 \mathrm{~V} 2^{\mathrm{T}}+\ldots \ldots .+\lambda \mathrm{rUrVr}^{\mathrm{T}}
$$

Where $r$ is the rank of matrix A. It is important to note that each singular value specifies the luminance of an image layer while the corresponding pair of singular vectors specifies the geometry of the image layer.

An important property of SVD based watermarking is that the largest of the modified singular values change very little for most types of attacks like transpose, flip, rotation, scaling and translation [8]. 


\subsection{Edge Detection}

Edge detection is one of the fundamental operations in computer vision with numerous approaches to it. In an historical paper, Marr and Hildreth introduced the theory of edge detection and described a method for determining the edges using the zero-crossings of the Laplacian of Gaussian of an image [9]. Haralick determined edges by fitting polynomial functions to local image intensities and finding the zerocrossings of the second directional derivative of the functions [10]. Canny determined edges by an optimization process and proposed an approximation to the optimal detector as the maxima of gradient magnitude of a Gaussian smoothed image [11]. Clark and Ulupinar and Medioni independently found a method to filter out false edges obtained by the Laplacian of Gaussian operator [12][13]. Bergholm introduced the concept of edge focusing and tracked edges from coarse to fine to mask weak and noisy edges [14].

Among the edge detection methods proposed so far, the Canny edge detector is the most rigorously defined operator and is widely used. The popularity of the Canny edge detector can be attributed to its optimality according to the three criteria of good detection, good localization, and single response to an edge. The Canny edge detector is widely used in computer vision to locate sharp intensity changes and to find object boundaries in an image. The Canny edge detector classifies a pixel as an edge if the gradient magnitude of the pixel is larger than those of pixels at both its sides in the direction of maximum intensity change [15].

\subsection{Discrete Wavelet Transform}

Wavelet domain is based on wavelet transformations. In wavelet domain which both time and frequency information are present. Wavelets are widely used in many fields because of their advantages like they offer simultaneous localization in time and frequency and they offer fast computation. DWT coefficient are divided into four components LL, LH, HL and $\mathrm{HH}$. LL is component with low frequency band and LH, HL and $\mathrm{HH}$ components are high frequency band. HVS are sensitive to low frequency band, so the watermark is inserted into high frequency band as changes in this band is not visible[16] .

The steps of using DWT are as following [17]:

- In this the original image is decomposed in various sub bands.

- DWT transformation, these sub bands can be decomposed to one, two or three level.

- The watermark is embedded in the sub band that is most suitable.

- After embedding watermark inverse transformation is performed and watermarked image is obtained. Fig 1. reveals the decomposition of 3-level DWT.

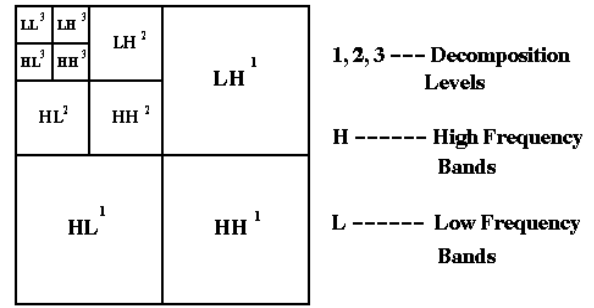

Fig 1: Decomposition of 3- level DWT

\section{PROPOSED METHOD}

A new method using edge detection and SVD-DWT based watermarking with edge detection is proposed which is robust under various attacks.

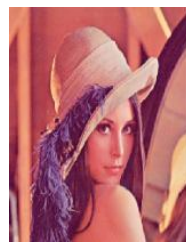

(a)

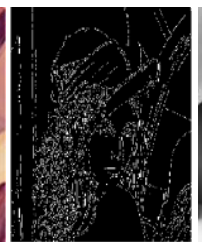

(b)

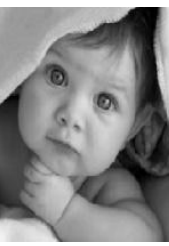

(c)

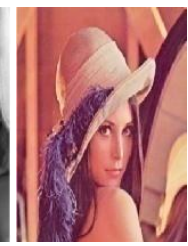

(d)
Fig 2 : (a) Original Image (b) Watermark1/Edge Image (c) Watermark2/ gray scale (d) Watermarked Image

\subsection{Watermarking embedding process}

The host color image has dimension of $512 \times 512$ pixels. The first watermark is edges of the host image and second watermark is grey scale image of size 512 x 512 pixels.

The embedding process is as follows:

1. Select the original host image

2. Generate the edge image from the original image and treat it as $1^{\text {st }}$ watermark.

3. Select the $2^{\text {nd }}$ watermark

4. Embed the $1^{\text {st }}$ watermark on one layer of original color image using SVD and it will generate new SVD matrix.

5. Embed the $2^{\text {nd }}$ watermark on another layer of original image using Discrete Wavelet transform.

6. Write and save the watermarked image which is generated from original host image in which two watermarks are hidden.

\subsection{Watermarking extracting process}

The extraction process is as follows:

1. Select the watermarked image.

2. Extract the $1^{\text {st }}$ watermark from one layer of host image by using new svd matrix and extract it by subtracting it from original matrix.

3. Extract the $2^{\text {nd }}$ watermark from another layer from host image using dwt .

4. Then select the original watermarks and compare them with the watermarks which are extracted.

5. Then calculate the different parameters required to compare them.

6. Watermarks can also be extracted in the same way if any attack is applied to the image.

With this algorithm the watermark can be embedded and extracted on the different layers of the host image.

\section{EXPERIMENTAL RESULTS}

The performance of proposed watermarking algorithm is explored using various experiments are calculated using different image such as Lena, Peppers , Cameraman of size $512 \times 512$.

For standard test 512 × 512 color images were used for studying the effects of imperceptibility and robustness of algorithm with scaling factor 0.1 . The imperceptibility and robustness are the properties that are evaluated for the 
proposed scheme. Imperceptibility means that the superficial quality of the original image should not be distorted even after presence of watermark image.

On the other hand, the robustness is the measure of the intentional and unintentional attacks. The Poisson signal-tonoise ratio (PSNR), mean square error (MSE) and Bit Error Rate (BER ) is used to measure the quality between original image and watermarked image.

The proposed scheme was tested against various image processing attacks: Gaussian noise, histogram, rotation, salt and pepper, speckle noise, Poisson noise, median filter and Jpeg compression. Table 1 contains the PSNR, BER, Correlation Coefficient (CC) and MSE after embedding the watermark image. Table $2 \& 3$ shows the PSNR, BER, CC and MSE after extracting the watermark.

Table 1 - CC, PSNR, MSE and BER after embedding the watermark in different images:

\begin{tabular}{|c|c|c|c|c|c|}
\hline IMAGE & & CC & PSNR & MSE & BER \\
\hline Lena & Penguin & 0.9995 & 50.6503 & 0.5598 & 0.0197 \\
\hline Peppers & 0.9998 & 50.7240 & 0.5504 & 0.0197 \\
\hline Baboon & & 0.9994 & 50.6505 & 0.5598 & 0.0197 \\
\hline
\end{tabular}

Table 2 - CC, PSNR ,MSE and BER after extracting watermark from different images with watermark 1 :

\begin{tabular}{|c|c|c|c|c|}
\hline Image & \multicolumn{4}{|c|}{ Watermark 1 } \\
\hline & CC1 & PSNR1 & MSE1 & BER1 \\
\hline Lena & 0.99901 & 87.0014 & $\begin{array}{c}1.2970 \mathrm{e}- \\
004\end{array}$ & 0.0115 \\
\hline Penguin & 0.99899 & 87.2647 & $\begin{array}{c}1.2207 \mathrm{e}- \\
004\end{array}$ & 0.0115 \\
\hline Peppers & 0.99881 & 87.1311 & $\begin{array}{c}1.2589 \mathrm{e}- \\
004\end{array}$ & 0.0115 \\
\hline Baboon & 0.99892 & 83.6830 & $\begin{array}{c}2.7847 \mathrm{e}- \\
004\end{array}$ & 0.0119 \\
\hline
\end{tabular}

Table 3 - CC, PSNR ,MSE and BER after extracting watermark from different images with watermark 2.

\begin{tabular}{|c|c|c|c|c|}
\hline Image & \multicolumn{4}{|c|}{ Watermark 2 } \\
\hline & CC2 & PSNR2 & MSE2 & BER2 \\
\hline Lena & 0.995 & 36.6744 & 13.9842 & 0.0273 \\
\hline Penguin & 0.97404 & 35.1146 & 20.0273 & 0.0285 \\
\hline
\end{tabular}

\begin{tabular}{|c|c|c|c|c|}
\hline Peppers & 0.99234 & 36.3533 & 15.0575 & 0.0275 \\
\hline Baboon & 0.99501 & 36.6732 & 13.9881 & 0.0273 \\
\hline
\end{tabular}

\subsection{Performance Evaluation}

In order to check the similarity of the extract watermark and origin image, Correlation Coeffecient (CC) is used to detect the original watermark and extracted watermark.

In [1] almost every attract could be analyzed and proposed the robust region- adaptive dual image watermarking technique. It was not to deal with transform image. Moreover, region was randomly selected to hide the watermarks in it which are in less size than the original image. In the proposed scheme, both the watermarks are of same size as the original image. It can be seen that the proposed method could attain high quality extraction of watermark

For testing the images after various attacks, Lena image is subjected to attacks. Table 4 shows PSNR values after the extraction of watermarks when the image after applying various attacks. Fig. 3 depicts the effect of attacks on the visual quality of the Lena image.

Table 4 Comparison of Extracting Watermark Performance under Various Attacks

\begin{tabular}{|l|c|c|c|c|}
\hline \multirow{2}{*}{$\begin{array}{c}\text { Attack } \\
\text { Method }\end{array}$} & \multicolumn{2}{|c|}{$\begin{array}{c}\text { Existing Method } \\
\text { (Dacheng Xu et } \\
\text { al.,2013) }\end{array}$} & \multicolumn{2}{c|}{ Proposed method } \\
\cline { 2 - 5 } & $\begin{array}{c}\text { First } \\
\text { wmk }\end{array}$ & $\begin{array}{c}\text { Second } \\
\text { Wmk }\end{array}$ & $\begin{array}{c}\text { First } \\
\text { wmk }\end{array}$ & $\begin{array}{c}\text { Second } \\
\text { wmk }\end{array}$ \\
\hline $\begin{array}{l}\text { Salt \& } \\
\text { pepper noise }\end{array}$ & 0.4573 & 0.8710 & 0.98679 & 0.98566 \\
\hline $\begin{array}{l}\text { Gaussian } \\
\text { noise }\end{array}$ & 0.4271 & 0.4055 & 0.98628 & 0.57556 \\
\hline $\begin{array}{l}\text { Rotate } \\
\text { image 45 }\end{array}$ & 0.5227 & 0.4573 & 0.9868 & 0.31861 \\
\hline $\begin{array}{l}\text { Histogram } \\
\text { equalization }\end{array}$ & 0.3896 & 0.4253 & 0.98578 & 0.086498 \\
\hline $\begin{array}{l}\text { Median } \\
\text { filter }\end{array}$ & 0.7227 & 0.4060 & 0.80999 & 0.77287 \\
\hline Jpeg attack & 1.0913 & 0.4197 & 0.88436 & 0.78767 \\
\hline
\end{tabular}

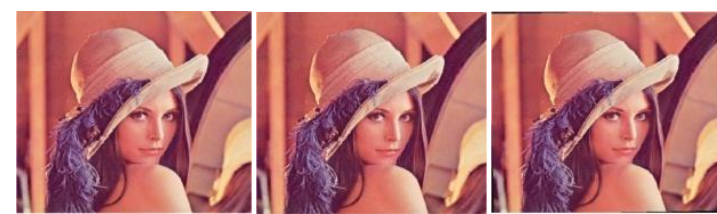

(a)Salt \&pepper noise (b)Gaussian Noise(c) Rotate Image 45

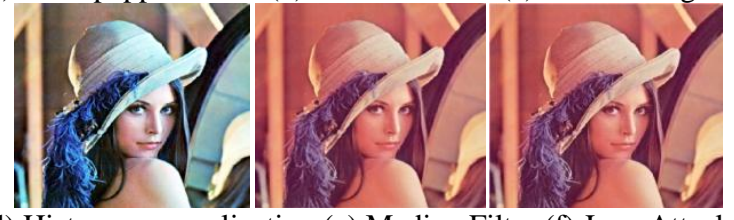

(d) Histogram equalization (e) Median Filter (f) Jpeg Attack

Fig 3 : Watermark Lena image with various attacks. 


\section{CONCLUSION}

In this paper, edge detection and SVD-DWT is used for watermarking for the purpose of high authentication of original image and security of image. The proposed Dual image watermarking algorithm based on SVD-DWT and Edge detection has improved the robustness and imperceptibility. It could efficiently protect the both watermark information and original data. Experimental results showed that the proposed algorithm was more robust to various attacks. In the future work, we should improve the response of various attacks.

\section{REFERENCES}

[1] Dacheng $\mathrm{Xu}$, Bialiang Li,Minghui Shi, "Dual Image Watermarking Algorithm Based on Image Segmentation And Linear Weighted Function Codec Technology", IEEE conference, pp 1367-1371, 2013.

[2] R.G. Schyndel, A. Tirkel, and C.F Osborne, -A Digital Watermarkll, Proceedings Of IEEE International Conference On Image Processing, ICIP-1994, pp. 86-90, 1994.

[3] Ramandeep kaur and Sonika Jindal "Robust Digital Image watermarking in High Frequency Band Using Median Filter Function Based on DWT-SVD" accepted in IEEE fourth international conference 2014

[4] Zude Zhou, Bing Tang and Xinhua Liu, "A Block -SVD Based Image Watermarking Method." IEEE Trans on Intelligent Control and Automation, Vol. 2, pp 1034710351, 2006.

[5] Gaurav Bhatnagar, Balasubramanian Raman, K. Swaminathan "DWT-SVD based Dual Watermarking Scheme” IEEE ,pp 526-531,2008.

[6] Kapre bhagyashri, S., Joshi, M.Y. “ Robust Image Watermarking Based on Singular Value Decomposition and Discrete Wavelet Transform" proceedings of IEEE $3^{\text {rd }}$ international conference, vol 5, pp 337-341, 2010.

[7] Praful Saxena, Shanon Garg and Arpita Srivastava, "DWT-SVD Semi- Blind Image Watermarking Using
High Frequency Band", $2^{\text {nd }}$ International Conference on Computer Science and Information Technology (ICCSIT'2012) Singapore April 28-29, 2012.

[8] Rajani, A. Dr. T. Ramashri “ Image Watermarking Algorithm Using DCT SVD and Edge Detection Technique" International Journal Of Engg. Research \& Applications(IJERA) ISSN: 2248-9622, vol 1, issue 4, pp: 1828-1834.

[9] D. Marr, E. Hildreth, Theory of edge detection, Proc Roy. Soc. London B-207 (1980) 187-217.

[10] R. Haralick, Digital Step Edges From Zero Crossing Of Second Directional Derivatives, IEEE Trans. Pattern Anal. Mach. Intell. 6 (1984) 58-68.

[11] J. Canny, A computational Approach To Edge Detection, IEEE Trans. Pattern Anal. Mach. Intell. 8 (6) (1986)679698.

[12] J.J. Clark, Authenticating Edges Produced By Zero Crossing Algorithms, IEEE Trans. Pattern Anal. Mach. Intell. 11 (1)(1989) 43-57.

[13] F. Ulupinar, G. Medioni, Refining Edges Detected By LoG Operator, Computer Vision, Graphics, Image Process.51 (1990) 275-298.

[14] F. Bergholm, Edge Focusing, IEEE Trans. Pattern Anal.Mach. Intell. 9 (1987) 726-741.

[15] Lijun Ding, Ardeshir Goshtasby “ On the Canny Edge Detector" Journal Of The Pattern Recognition Society, pp 721-725, 2001.

[16] Z. Zhuancheng, Z. Dianfu, Y. Xiaoping, "A robust Image Blind Watermarking Algorithm Based On Adaptive Quantization Step In DWT", Journal of Image and Graphics 11 (6) (2006) 840-847.

[17] Divjot kaur Thind, Sonika Jindal "Review of Watermarking And Its Techniques", ICRTEDC, Vol 1, Spl. Issue 2, May 2014. 